\title{
Review
}

Acta Cytologica 2013;57:221-232

DOI: $\underline{10.1159 / 000350718}$
Received: March 17, 2013

Accepted after revision: March 18, 2013

Published online: April 25, 2013

\section{Telepathology in Cytopathology: Challenges and Opportunities}

\author{
Brian T. Collins \\ Department of Pathology and Immunology, Washington University in St. Louis School of Medicine, \\ St. Louis, Mo., USA
}

\section{Key Words}

Telepathology · Telemedicine · Cytopathology · Rapid on-site evaluation - Fine needle aspiration biopsy

\begin{abstract}
Objective: Telepathology in cytopathology is becoming more commonly utilized, and newer technologic infrastructures afford the laboratory a variety of options. The options and design of a telepathology system are driven by the clinical needs. This is primarily focused on providing rapid onsite evaluation service for fine needle aspiration. Study Design: The clinical requirements and needs of a system are described. Available tools to design and implement a telepathology system are covered, including methods of image capture, network connectivity and remote viewing options. Results: The primary telepathology method currently used and described involves the delivery via a network connection of a live video image to a remote site which is passively viewed by an internet web-based browser. By utilizing live video information and a voice connection to the on-site location, the remote viewer can collect clinical information and direct their view of the slides. Conclusions: Telepathology systems for use in cytopathology can be designed and implemented with commercially available infrastructure. It is necessary for the laboratory to validate the designed system
\end{abstract}

and adhere to the required regulatory requirements. Telepathology for cytopathology can be reliably utilized by adapting existing technology, and newer advances hold great promise for further applications in the cytopathology laboratory.

Copyright $\odot 2013$ S. Karger AG, Basel

\section{Introduction}

Telepathology is increasingly being utilized in the cytopathology laboratory. The need is driven by a variety of internal and external factors and requirements. Rapid onsite evaluation (ROSE) is a service that the cytopathology laboratory provides for patients and their physicians. The advantages of immediate on-site evaluation include assurance of fine needle aspiration (FNA) biopsy adequacy, the ability to triage the specimen, shorten the length of the procedure, help guide and direct the biopsy in real time, and overall assist in managing the patient procedure with the performing clinician at the point of care. Those who work in the cytopathology laboratory recognize the value to patient care that the ROSE service provides. Additionally, the clinicians who perform the procedures also recognize the importance of ROSE for their patients and how it helps to guide the performance of their procedures.

\section{KARGER}

E-Mail karger@karger.com www.karger.com/acy
(C) 2013 S. Karger AG, Basel

0001-5547/13/0573-0221\$38.00/0
Correspondence to: Dr. Brian T. Collin

Department of Pathology and Immunology

Washington University in St. Louis, Campus Box 8118, 660 South Euclid Avenue St. Louis, MO 63110-1093 (USA)

E-Mail bcollins@path.wustl.edu 
FNA biopsy is performed in a wide variety of anatomic organs and areas within the body and by a varied mix of clinicians. These commonly include the thoracic cavity via endobronchial ultrasound (EBUS) guidance performed by pulmonologists and thoracic surgeons, thoracic and abdominal cavity including the liver, stomach, pancreas, spleen and adrenal glands via endoscopic ultrasound (EUS) guidance performed by gastroenterologists, as well as head and neck, thyroid, thoracic and abdominal locations via ultrasound (US) and CT-guided methods performed by otolaryngologists, endocrinologists and radiologists. As such, the need arises in an assortment of situations within hospital and outpatient settings.

Based on these patient care needs and performing clinician requests, the cytopathology laboratory endeavors to provide the ROSE service to all their patients and health care partners who require it. As more patients need it, the cytopathology laboratory is faced with the challenge of providing a labor-intensive service to multiple health care providers at multiple times during the day at multiple locations. This can multiply into a problem when there are insufficient resources or staffing to cover the numerous clinical requests.

When any service is provided to clinicians, they come to rely on it and depend on it being available when requested. The nature of EUS, EBUS, CT and US FNA biopsy results in an inability to schedule specific procedure times, and therefore, the cytopathology laboratory must often provide the ROSE service in an 'on-call' manner. This makes it very difficult to adequately staff and cover all the patients and physicians who require the service. This can result in a 'feast or famine' service where multiple cases occur at the same time or overlap and at other times when they occur singly. Time commitment for the ROSE service is significant. It is provided by being in the room or suite where the FNA biopsy occurs, which is physically distant from the cytopathology laboratory, and requires travel time to and from the site, as well as the time required to review slides and wait for additional FNA biopsies, as necessary. This limits the health care professionals involved to a single procedure at any one time.

In addition to overlapping or concomitant procedures, there can be physical and distance limitations. Radiology, endocrinology and endoscopy suites can be situated in locations far removed from the cytopathology laboratory or in buildings separate from the hospital-based laboratory. These volume, timing and distance issues can be roadblocks and bottlenecks for providing immediate onsite evaluation.
Telepathology provides the means to help improve the delivery of the ROSE service for patients and clinicians and alleviate some of the challenges and limitations based on volume and distance constraints. A variety of institutions and practices have described their experience with telepathology in the cytopathology laboratory [1-26]. The aim of this article is to discuss the concepts and goals of telepathology, review the infrastructure necessary for implementation, and cover the necessary validation and regulatory requirements.

\section{Concepts and Goals}

Telepathology as a specific term has multiple meanings, depending on its intended usage and specific infrastructure. The ultimate goal of a 'telepathology' system is to deliver the real-time data (images) to the individual (eyeballs) who has the training and expertise to provide an interpretation at a separate, remote location. In general, telepathology involves the ability to capture image(s) of visually based pathologic material at one site and deliver them over distance to another separate site. This requires a camera system to capture images, a way to store images and a method to deliver and view the images. These will each be covered briefly as individual concepts. There is no 'one size fits all' solution. Each cytopathology laboratory will have different needs and different health care paradigms which require individual customization in order to create the optimal telepathology system best suited to the unique practice demands. No two laboratories are alike and no two telepathology systems will be alike either. There is no standard of care system or single telepathology model. All configurations and potential systems described are currently commercially available and do not require the creation of unique one of a kind devices. The components necessary are 'off the shelf and currently available. There is a developed medical literature covering telepathology and the technologic infrastructure in its use, as well as resources and professional societies dedicated to telepathology and pathology informatics [27-47].

\section{Camera System}

Most cameras will be CCD based and color capable with an attachment to a phototube on a microscope. There are adaptors which allow hand-held cameras and smart phones to capture images through the ocular eye- 
pieces. These cameras are typically capable of both still image capture (single snap shot picture) and dynamic live video-style moving images. Whole slide image (WSI) devices scan and capture an entire pathology slide. A wide variety of vendors offer cameras and adaptor options which are suitable for usage in telepathology.

\section{Image Storage System}

Images may be captured as individual snap shots at the originating site. These are saved or stored on a physical device such as the memory of the camera or on a computer when the camera is connected to a PC. Video-style moving images can be sent/'streamed' live or captured as a standalone video file for delivery. WSI scans are stored on a local network for remote access or can be saved as a single large file for delivery.

\section{Method of Delivery}

Once the image(s) have been captured and stored at the originating site, they need to be delivered to the remote location. The camera/computer device which captures the images needs to be connected to a computer network in some manner. This infrastructure is usually in place for outpatient and inpatient hospital health care facilities; however, individual rooms/suites might need additional access points added. The network connection can be via a wireless Wi-Fi system. Wi-Fi wireless systems can present bottlenecks due to their inherent ability to be able to handle only limited amounts of data when transmitted images require a high bandwidth connection. A physical hard-wired Ethernet network is commonly present in most buildings, and this will allow communication to the network and accommodate high volume data transfers. Static images can be delivered via e-mail and live video is delivered directly to the distant site.

\section{Viewing Images}

It is necessary to have a device for viewing the images captured and delivered from the originating location. This is usually a network-connected PC with visual video monitor. Newer devices such as smartphones and tablets $\left(\mathrm{iPad}^{\mathrm{TM}}\right)$ have the potential to provide high-resolution image viewing and allow for more individual user mobility than a fixed location static hard-wired PC system.

Telepathology in Cytopathology

\section{Procedure Communication}

Utilizing telepathology for the ROSE service occurs in real time, and being able to communicate between the originating and remote site is important. It is also necessary to be College of American Pathologists (CAP) telepathology checklist compliant, which requires the remote site to be able to positively identify the patient and obtain the necessary medical information to provide the pathology interpretation. This is best performed by verbal communication which can be accomplished by a telepathology connection between the two sites. This is where the 'tele' in telepathology originates but it has moved far beyond a sole dependence on the telephone data system. A VoIP (voice over IP) or computer microphone transmitted between the two sites can also accomplish a verbal connection for a live real-time procedure.

\section{Health Insurance Portability and Accountability Act Considerations}

The capture and delivery methods for a telepathology system should be designed and executed by following procedures which assure protection of Health Insurance Portability and Accountability Act (HIPAA)-related patient information. It is important to work with the information technology staff of the health care systems to ensure that appropriate procedures are in place during the transfer of information. These procedures should be secure, confidential and conform to HIPAA patient confidentiality needs. These are included in the CAP laboratory accreditation requirements detailed below.

\section{Bottlenecks}

ROSE service for FNA biopsy is a time-limited procedure which occurs while patients are subject to varying degrees of sedation and while they have endoscopes or large needles placed within the thoracic cavity or abdomen. As such, it is important to collect and deliver images as quickly as possible in the highest quality. These relatively complex systems can have a variety of data 'narrow' points which can slow the overall service. These include slow image acquisition, network data transfer limitations which slow communication, and inadequate or suboptimal viewing experiences due to technical limitations of the system. It is important to be aware that a failure in one link of the chain in a telepathology system can 
affect the entire service and be a cause for overall system failure. It is necessary to consider all individual steps in the process and how the cytopathology laboratory can implement and leverage their resources to design and deploy the best telepathology system possible.

\section{Common and Current Systems for Telepathology}

A few of the more prevalent options for a telepathology system in cytopathology will be discussed, including the predominant infrastructure commonly used and described in the cytopathology literature.

\section{Static Image Capture System}

A microscope with a camera attached can take static still images. These can be saved as individual files and then transmitted via e-mail to a remote individual. Before the advent of newer camera systems capable of live video streaming and more robust large bandwidth data networks, individual image capture images were one of the early methods of telepathology since it was amenable to the systems available at the time. One of the major limitations of a static image capture system is the selective and narrow scope of pathology review based on still images selected by the originating site. The advantages include a basic infrastructure requirement. There is considerable dependence on the image selection made by the operator at the originating site, who must assure that they provide representative high-quality images which illustrate the salient morphologic features of the slide(s).

\section{Live Video System}

The most commonly described telepathology system for the cytopathology ROSE service involves a live motion video system. In its basic design, this involves a microscope which has a device attached to it with a camera capable of generating video. The microscope is located in or close to the FNA biopsy procedure site, is connected to the network and has a method for delivering the video image present in the microscope to the remote site. All of these processes and configurations involve a 'passive' viewing experience for the remote site. The operator at the originating site where the FNA biopsy is being performed controls the microscope and has primary physical control over the viewing experience. The remote site distant from the FNA biopsy cannot independently or physically control the movement of the microscope or the image. The remote site can verbally communicate with the on-site operator to move and adjust the viewing fields based on their observations of the live video image. This 'team' approach is vitally important to successfully delivering a telepathology ROSE service.

Since the remote viewing site is passive, there is a significant degree of reliance placed on the operator who is at the FNA biopsy point of care. The experience of the on-site telepathology operator and their rapid and accurate locator skills make the process much easier for the remote site.

The telepathology service is not a screening procedure in the traditional sense of a slow deliberate back and forth movement to cover the entirety of the slide. The ROSE service is time sensitive with patients either under anesthesia or waiting with a large needle placed within a deep organ. A telepathology ROSE service needs to closely approximate the timely evaluation of slides which would be performed with a single individual at the site viewing the slides.

\section{Team Approach}

There are two main members of the team and these are the on-site telepathology operator and the remote-site telepathology viewer.

\section{On-Site Telepathology Operator}

The individual who operates the telepathology system on site is critically important to its success. A slow methodical and mechanical full-slide medium-power screening is unacceptable due to the excess time involved in identifying the 'cells of interest'. Therefore, the on-site operator needs to be a health care professional who has experience with morphologic observations and pathologic diagnosis. These would include a cytotechnologist, a pathology resident, a cytopathology fellow and a pathologist. It would not include a nurse, radiology technician, endoscopy nurse or non-pathology physician. These individuals do not have the experience or ability to view and direct the live image to the areas of interest necessary for interpretation by the remote viewer. As required by CAP and further detailed below, the correct patient must be identified and communicated to the remote viewer and the pertinent clinical information must be delivered before the case is reviewed.

\section{Remote-Site Telepathology Viewer}

The remote-site health care professional relies on the on-site telepathology operator to view and direct the live video images to present a representative view of the 
slide(s). In the various systems available, some minor adjustments by the remote viewer are possible, including changes in resolution and screen size. However, these are relatively minor in the overall telepathology experience, whereas the ability to have the 'cells of interest' quickly located and seen is far more important. This assumes that the system designed can provide a good resolution image of the FNA biopsy smear material. A low-power resolution $(320 \times 240$ screen image resolution) is limited in being able to provide the detail necessary for interpretation, and a $640 \times 480$ screen image resolution is significantly better at illustrating the morphologic features present and necessary for interpretation.

\section{Low- and High-Power Objective Viewing}

The remote viewer has a different experience with 'motion' viewing and stable higher-power 'static' viewing. 'Motion' viewing involves movement around the slide with a lower-power objective and provides the remote site with a general 'sense' of the slide cellularity and low-power smear pattern, which are important features of adequacy and interpretation. This 'motion' viewing can be performed at lower screen image resolution. In fact, in some systems, a low-power objective motion viewing can be 'halting' and 'jerky' if the camera capture screen image resolution is set to a higher resolution $(1,280$ $\times 960$ screen image resolution). Therefore, the viewing experience for low-power objectives can be better without the demands of high-resolution screen image.

Once a group or area has been identified for closer examination, this can be framed by a higher-power objective $(\times 20-40)$ and then viewed in a higher-resolution screen image with less 'jerking' motion and better visualization of the subtle details necessary for interpretation. $40 \times$ immediate slides (typically modified Wright-Giemsa stain) can have a 'fuzzy' appearance because they lack a cover slip. This is true for the on-site experience which is also reflected by the telepathology system. Using a 'wet' coverslip method can help to improve the clarity of the image captured by the higher-power microscope objectives which is then able to be conveyed via the telepathology system.

\section{Live Video Systems}

\section{Nikon System}

The Nikon ${ }^{\mathrm{TM}}$ imaging system is commercially available and involves a combination of a digital microscope

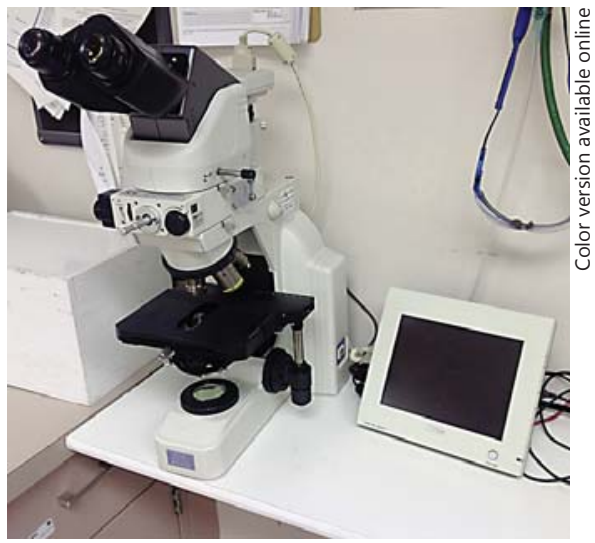

Fig. 1. Nikon DS-L2 hardware configuration showing the standalone viewer connected to the microscope camera. The controller can send out a variety of live images as well as capture still images (Nikon Digital Sight Camera Controller DS-L2). Used with permission.

camera system (DS-Ri1) and stand-alone microscope control unit (DS-L3; http://www.nikoninstruments.com/ Products/Cameras/Controllers). This system does not require a dedicated PC. The Nikon DS-L3 control unit can send static images and live video feeds from the FNA biopsy site simultaneously on the DS-L3 screen and a projector (DVI-1), PC monitor (USB) and network connection (LAN/Ethernet). This allows the image that is being captured to be viewed in the FNA biopsy room, so that the operator can see what is being sent to the remote site (fig. 1). The network connection from the Nikon camera controller is 10Base-T/100Base-TX compatible. The remote user accesses an HTTP/IP address via a web browser (e.g., Internet Explorer, Firefox), and the live image is streamed to the desktop PC. The size of the image and the resolution of the live image can be changed to fit the desktop and improve image screen resolution. The on-site operator and remote viewer communicate in real time via a voice-based telephone connection.

Once set up, the system is very user friendly and requires minimal effort to use. Once the DS-L3 is turned on, it captures the live image seen in the microscope and makes it available to the remote site. This makes it easy to use, provides a simple user interface and delivers a stable consistent live video image to the remote viewer. The remote viewer can bookmark the IP/web site address, click on this bookmark and the first screen 'room' choices appear (fig. 2). After clicking the appropriate 'room' choice, the live image loads in the web browser (fig. 3). If there is a technical problem or absence of image, restarting the 


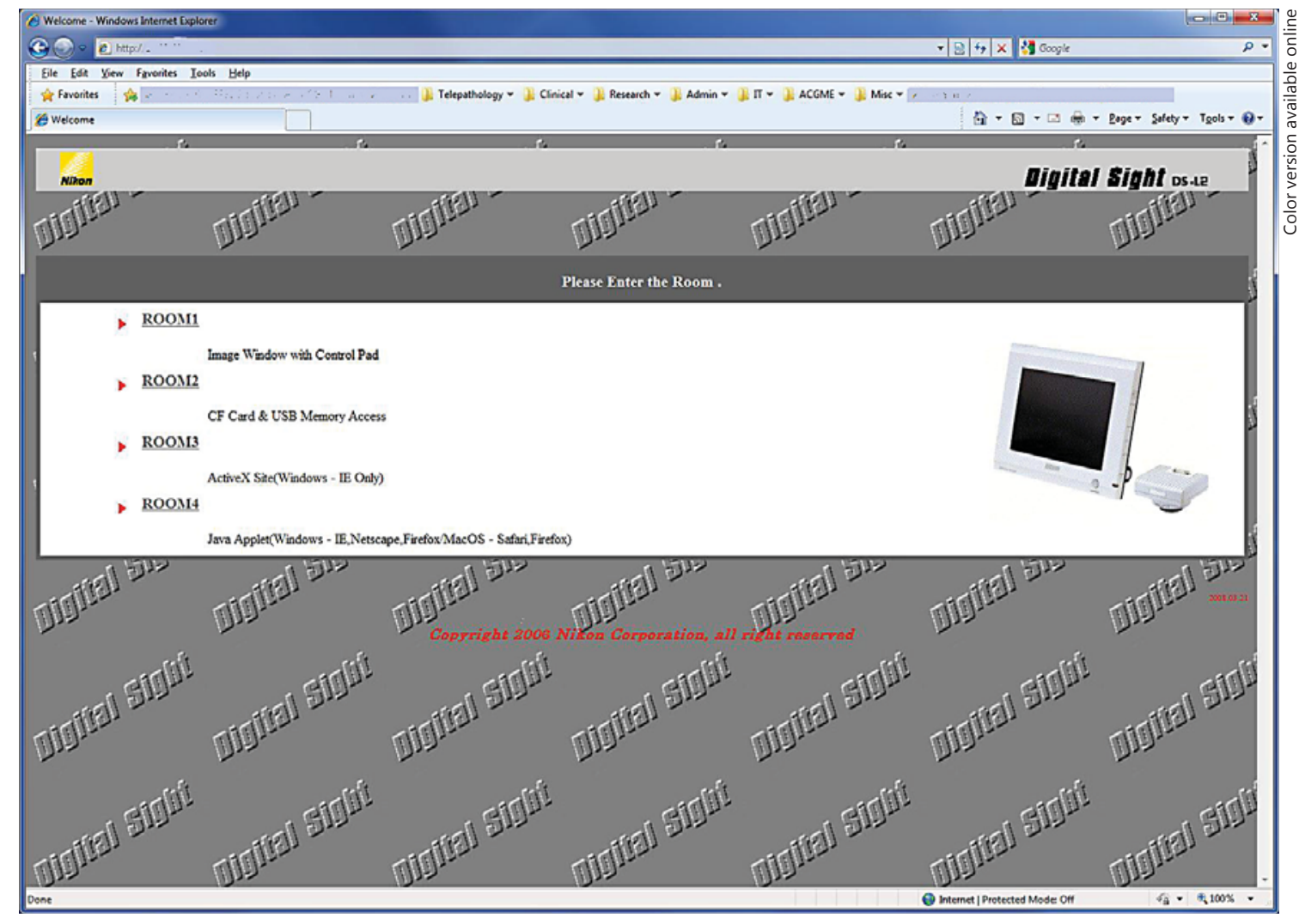

Fig. 2. Screen capture of the web-based Internet web browser (Internet Explorer, Windows System) which the remote viewer accesses in order to select a specific live video feed (Screen capture, PC Windows System, Internet Explorer, Nikon Digital Sight Camera Controller Interface).

DS-L3 (which only takes a few moments) and restarting the remote viewer browser usually resolves the problem. The DS-L3 control unit is small and takes up very little space, as well as providing a direct viewing option next to the microscope. It does not require a separate stand-alone PC. While a live video image cannot be captured and illustrated for a print journal, illustrations of the screen appearance of live video are demonstrated by screen capture (fig. 4, 5).

\section{Olympus System}

The Olympus ${ }^{\mathrm{TM}}$ imaging system is commercially available and involves the combination of a digital microscope camera (DP-X) and a stand-alone PC with Olympus image software (CellSens ${ }^{\circledR}$; http://www.olympusamerica. com). The camera connects to the PC via a USB connector or a computer card. After turning on the microscope, the PC attached to the camera must be booted up and Windows loaded. CellSens only works in the Windows environment. After the Windows operating system boot up and log in process, the CellSens Olympus image software must be started. Within the software window, the live video image can be viewed. In order to provide remote live video viewing, the CellSens image software needs to have a paid upgrade from the basic provided package in order to provide NetCam capability. The NetCam option needs to be checked/clicked to begin the process of a web-based http/IP video image feed. The remote viewer opens the predesignated IP/http site in an internet-based web browser. The image resolution can be adjusted by the web browser interface, and the dimensions of the image in the web browser can be adjusted. The onsite operator and remote viewer communicate in real time via a voice-based telephone connection.

The Olympus system requires a dedicated PC and involves a more detailed and complicated start process. This requires a PC bootup via Windows, manually starting the CellSens image software, and then manually enabling the NetCam video feed. 
Fig. 3. Screen capture of a live image seen at a remote viewing site on a PC monitor via a web-based browser. This shows a modified Wright-Giemsa stain direct smear from an EBUS-guided FNA of paratracheal lymph node with extensive necrosis, but lacking viable tumor cells (Screen capture, PC Windows System, Internet Explorer, Nikon Digital Sight Camera Controller Interface).

Fig. 4. Screen capture of a live image seen at a remote viewing site on a PC monitor via a web-based browser. This shows a modified Wright-Giemsa stain direct smear from an EBUS-guided FNA of paratracheal lymph node with non-small cell carcinoma at $20 \times$ objective view (Screen capture, PC Windows System, Internet Explorer, Nikon Digital Sight Camera Controller Interface).
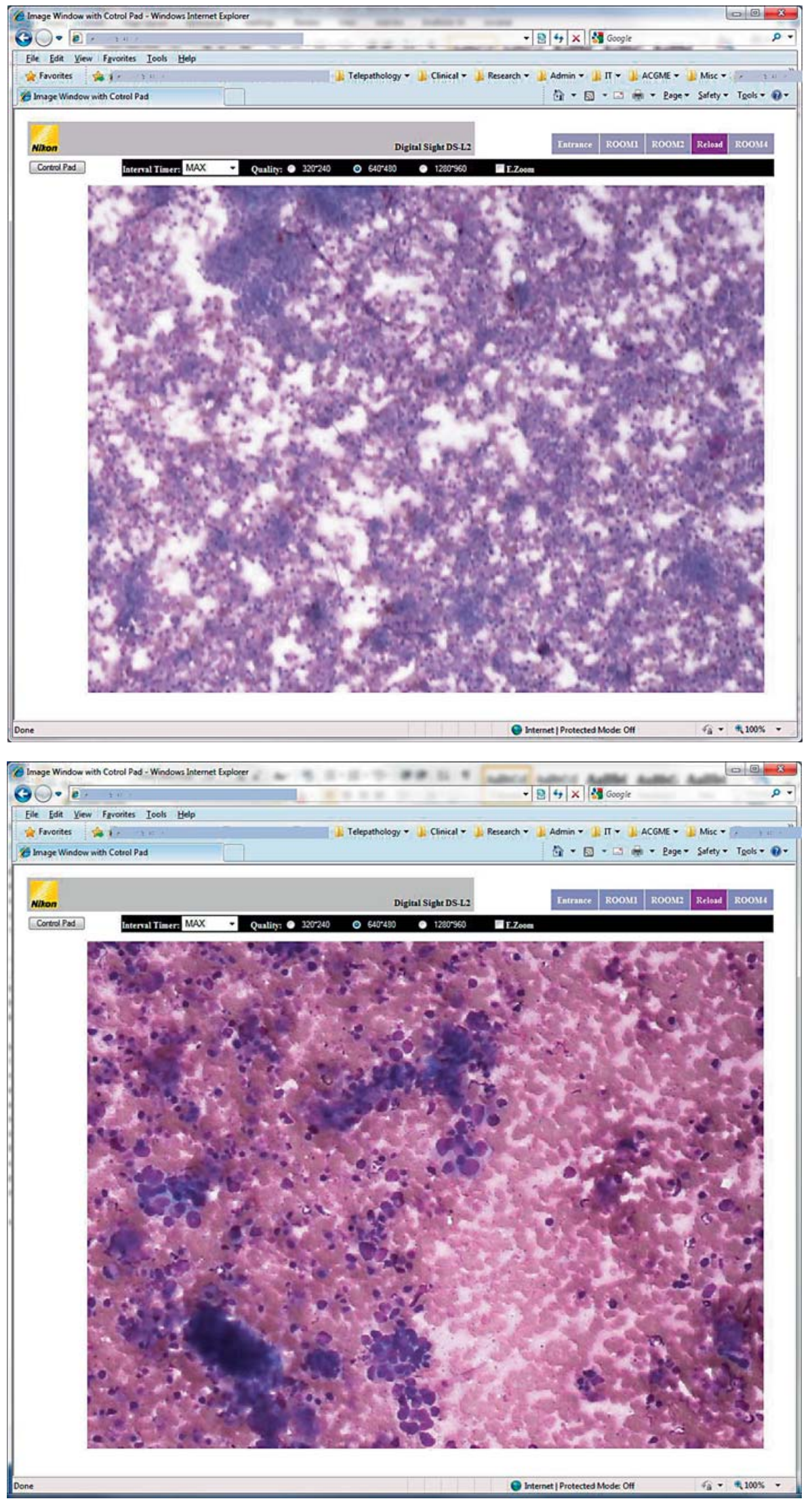
Fig. 5. Screen capture of a live image seen at a remote viewing site on a PC monitor via a web-based browser. This shows a modified Wright-Giemsa stain direct smear from an EBUS-guided FNA of paratracheal lymph node with non-small cell carcinoma at $20 \times$ objective view from the same case as in figure 4, providing enough detail and volume for an adequacy assessment (Screen capture, PC Windows System, Internet Explorer, Nikon Digital Sight Camera Controller Interface).

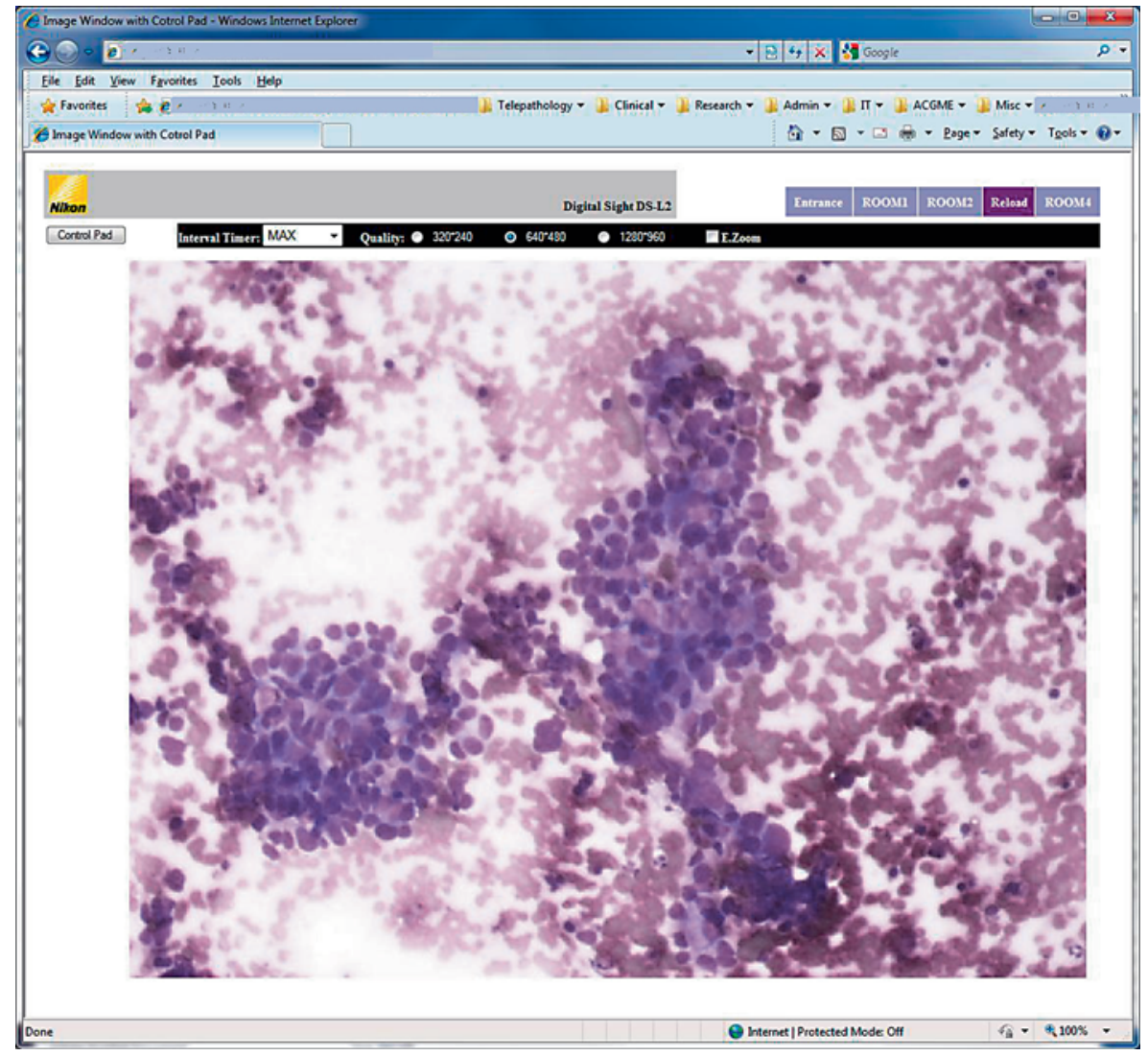

There are hardware and software differences between the Nikon and Olympus systems, and these differences are best judged by the needs, resources and demands of the unique health care environments in which they would be utilized. Both are capable of delivering a live microscopy video feed to a remote site viewed by a web browser via a network connection.

The overall cost of these systems will depend on a wide variety of factors. In some instances, pre-existing resources can be utilized (microscopes, cameras, network connections, PC) and integrated with newer resources (e.g., camera controller, image software). Depending on the system used and the resources available, the cost can be from USD 5,000 to 25,000. In most health care environments and laboratories, implementing a telepathology system requires an investment in order to provide the service.

\section{iPhone $^{T M}$ Adapter System}

Smart phones (iPhone, iPod ${ }^{\mathrm{TM}}$ Touch, Android-based phones, and others) are widely available and have built-in high-resolution (5 megapixel) cameras capable of still im- age capture and live video images. They have the ability to connect to network systems via a wireless method (WiFi or cellular network). Adaptors are available (http:// www.skylightscope.com/) which allow the smart phone to be connected to the microscope eyepiece. This permits the smart phone built-in camera to view the image in the ocular eyepiece. Static images can be captured and sent via wireless connection to an e-mail address. Live image feeds can be sent wirelessly utilizing the on-site smart phone apps/software (FaceTime ${ }^{\mathrm{TM}}$ for Apple, Skype ${ }^{\mathrm{TM}}$ for Android/Windows) to the remote viewer. The Apple environment allows for the live image to be delivered to any Apple iOS device (iPhone, iPad, iPad-mini, iPod Touch) and Mac-based system with FaceTime software.

Advantages of the smart phone system include portability, no need for a fixed dedicated microscope camera, lack of need for a hardwired Ethernet connection, and comparable affordability. A simple system could be assembled for as little as a few thousand dollars. Challenges include providing a stable wireless system that can deliver the data necessary to the remote viewer with a high enough quality to provide meaningful interpretation. 


\section{System Validation}

As with any procedure or test used in a clinical laboratory, it should undergo a validation process. Implementation of a successful system that works as intended begins with thoughtful foresight and pre-implementation planning. For an effective telepathology ROSE system, the laboratory should determine the location(s) of the FNA biopsy procedures, on-site needs for the physical hardware and network connectivity, and how to best staff and deliver the images to the remote site for viewing. Once a system has been designed, the component parts to the complex system need to be assembled and there should be an assurance that each works as necessary and intended. Failure at any step or point in the process can result in overall system failure or an unreliable process which does not provide the patient care it is designed to deliver. Before the system is validated, the individuals who will participate in the process should be comfortable with how the system functions and gain some experience with its use. Telepathology systems require practice and hands on use by users, even those who are comfortable with technology and image systems. Telepathology systems do have early adoption learning curves that improve with experience and practice. Learning how to use a newly designed and implemented telepathology system at the same time as the validation process can present problems with performing a true system validation.

After design, construction and individual step and component authentication, the telepathology system should be validated in a manner which mirrors its use in a real clinical practice setting. Since it has not been validated for patient use, it should not yet be deployed or implemented for FNA biopsy procedures. Instead, previously performed cases can be selected and used to validate the system. These cases should reflect the common spectrum of diagnostic entities which would be encountered at the ROSE FNA biopsy and include those that are diagnostic and unsatisfactory, as well as those with a final designation of indeterminate or suspicious. Only the portion of an individual case that would be available at the time of the telepathology (such as the air-dried modified Wright-Giemsa-stained smears) should be used, and these should be presented in the order that would be seen in the actual telepathology ROSE service. The on-site operator for the validation process should have the requisition and the clinical history available at the time of the original FNA biopsy and provide it to the remote viewer. The ROSE FNA service is not used for final diagnosis; therefore, the validation process should determine and record the overall adequacy of the case (either one site or multiple sites), and this should be measured against the final case outcome. The case volumes for validation and concordance rates should be determined by the cytopathology laboratory based on the complexity of the process and how well the system works under the validation process. If the initial validation test underperforms, then specific system challenges or difficulties can be identified and adjusted to improve the performance of the system. It is useful to continue to monitor the performance of the telepathology system after it is introduced into the clinical service. This helps to identify potential areas of improvement and assure the continued performance of the system as expected and designed. CAP laboratory accreditation requires including the telepathology system in the ongoing quality management process.

None of these systems is Food and Drug Administration approved. They are designed for investigative and research utilization only. Any system adapted and implemented for clinical usage should be validated for use by the individual cytopathology laboratory, in accordance with their overall hospital and laboratory policies and along with local, state and national accreditation guidelines and applicable regulatory requirements.

\section{Training Process}

After the telepathology system has been validated, the health care professions who will use the system should be trained in its operation and use. The lessons learned from the design, implementation and validation should be shared with the end users. The ability to test and practice on the system should be available, and the cytopathology laboratory supervisor or medical director should be a knowledgeable resource for the education and instruction of the users.

There are two main responsibilities for the personnel involved in the telepathology system and these are the onsite operator and the remote viewer. The on-site operator needs to be knowledgeable about (1) the hardware components involved and their appropriate connections; (2) the use of the software for capture of the camera images; (3) the ability of how to trouble shoot and optimize the image resolution and camera focus; (4) the overall telepathology process and end point goals, and (5) troubleshooting common potential problems with the system and how to recognize and correct them quickly.

The remote viewer needs to be knowledgeable about (1) how to access the live image via the web-based browser; (2) 
manipulate the image screen resolution options available in the software to optimize the viewing experience; (3) how to verbally communicate with the on-site operator via voice-based telephone to obtain the necessary patient information and identification; (4) coordinating the adequacy assessment work flow, and (5) how to determine ROSE FNA biopsy adequacy with the telepathology system.

Each individual user who is going to perform the specific functions (on-site operator and remote viewer) should demonstrate competency before using the system in a clinical setting, and this competency should be documented by the cytopathology laboratory. The remote viewers who will determine adequacy for the telepathology system should each be subject to a number of unknown cases by which they should demonstrate an acceptable rate of correct FNA biopsy immediate classification.

\section{Regulatory Environment}

The CAP laboratory accreditation program provides some general guidelines for the use of telepathology in the laboratories which fall under their accreditation. These are continually updated and refined based on a wide variety of factors and influences. At this time, there are some major principles which the CAP requires for laboratory accreditation. The category of 'telepathology' is broad and applies to static, dynamic (video) and WSIs (Aperio ${ }^{\mathrm{TM}}$ ). Use of these modalities can apply to clinical use in primary diagnoses, frozen section diagnosis, second opinion, and ancillary techniques. For the use of telepathology in these circumstances, there are a variety of requirements. The method and system used should be appropriate for the intended clinical use. The remote reviewer (telepathologist) should ensure correct patient identification and have access to pertinent clinical information at the time of the case review. Procedures should be in place to address the training requirements of all users of the system and these should be documented. The telepathology system should be included in the laboratory quality management program and monitored in an ongoing manner. Procedures should be in place to ensure patient confidentiality and security in conformance to HIPAA.

\section{Future Trends}

Live remote video telepathology works well in delivering the ROSE service for FNA biopsy procedures. This has been demonstrated by multiple series performed at a variety of institutions each with their own unique systems and demands. The live video images can be delivered in real time with good image resolution to allow for accurate and reproducible adequacy evaluation. Nevertheless, there are challenges inherent in the system design as well as opportunities for improving the telepathology delivery system. Foremost among the challenges is the 'passive' nature of the process. The commonly deployed systems currently used involve a remote review that occurs in a passive manner where the remote reviewer cannot directly control or direct the image view. A two person system with verbal communication directly to the remote operator can be slow and cumbersome at times.

As pathologists and cytotechnologists, we are accustomed to 'driving' when we view a slide, and it can be frustrating not to control the viewing experience. WSIs (Aperio and other vendors) present an opportunity to capture an entire slide and deliver it for active review by the remote site. This overcomes the impediment of passive control by the remote site. Nevertheless, there are challenges with this method. It takes time to scan an individual single slide (3-10 $\mathrm{min}$ ), and the delay can lengthen the procedure and limit the value of the ROSE service. An EUS FNA biopsy often has more than just a few immediate evaluation slides, and the slower process WSI time can be a limiting factor in its use since it could potentially take $30 \mathrm{~min}$ to scan 3 slides. WSI scanning does not do as well at capturing depth of field views (z stack) for thick or complex groups which are important in cytopathology evaluation. As the WSI technology improves and advances, it holds great promise for being able to deliver high-quality, accurate and timely scans of slides to a remote viewing site for immediate (and potentially even final) evaluation.

\section{Conclusion}

The goals of the telepathology service for cytopathology are the same as the goals for on-site ROSE. It is to provide an immediate evaluation of adequacy for the performing clinician, to only perform as many passes and sites as necessary, to assist in obtaining an adequate sample, to assure that the appropriate material is collected for final FNA biopsy diagnosis and to help to triage the biopsy as necessary for appropriate ancillary studies (flow cytometry, culture, molecular diagnostics, immunohistochemistry). The on-site ROSE service is not a final diagnosis and neither is the telepathology technique intended to provide a final interpretative FNA biopsy diagnosis. 
There are probably as many systems in use or possible as there are stars in the sky. The intent of this discussion is to detail the reasons and needs for telepathology in the cytopathology laboratory, and based on those needs, to discuss the commonly reported and commercially available systems in use and available. No doubt there are other potential solutions or novel methods of solving the current telepathology challenges; however, these would be outside the scope of our experience and knowledge. Since this is a new and emerging field with great potential for the further enhancement of patient care and improved efficiency in the utilization of health care resources, labo- ratories who have successfully implemented systems are encouraged to share their experience with the cytopathology community. In the future, telepathology is likely to be more commonly utilized in the cytopathology laboratory, and a better understanding of the underlying choices and clinical service needs will help laboratories to implement and adapt the technology for their patients.

\section{Disclosure Statement}

The authors have no financial disclosures to report.

\section{References}

1 Hudson J, Murray B, Chen A, Collins B, Wang J: Telepathology assisted immediate assessment of EBUS-FNA has equivalent accuracy to cytopathologists on-site examination. Mod Pathol 2013;26:92A.

- 2 Tsilalis T, Archondakis S, Meristoudis C Margari N, Pouliakis A, Skagias L, Panayiotides I, Karakitsos P: Assessment of static telecytological diagnoses' reproducibility in cervical smears prepared by means of liquidbased cytology. Telemed J E Health 2012;18 516-520.

3 Marotti JD, Johncox V, Ng D, Gonzalez JL, Padmanabhan V: Implementation of telecytology for immediate assessment of endoscopic ultrasound-guided fine-needle aspirations compared to conventional on-site evaluation: analysis of 240 consecutive cases. Acta Cytol 2012;56:548-553.

-4 Kumar N, Busarla SV, Sayed S, Kirimi JM, Okiro P, Gakinya SM, Moloo Z, Sohani AR: Telecytology in East Africa: a feasibility study of forty cases using a static imaging system. J Telemed Telecare 2012;18:7-12.

5 Khurana KK, Rong R, Wang D, Roy A: Dynamic telecytopathology for on-site preliminary diagnosis of endoscopic ultrasoundguided fine needle aspiration of pancreatic masses. J Telemed Telecare 2012;18:253-259.

6 Khurana KK: Telecytology and its evolving role in cytopathology. Diagn Cytopathol 2012;40:498-502.

-7 Heimann A, Maini G, Hwang S, Shroyer KR, Singh M: Use of telecytology for the immediate assessment of CT guided and endoscopic FNA cytology: diagnostic accuracy, advantages, and pitfalls. Diagn Cytopathol 2012;40: 575-581.

-8 Goyal A, Jhala N, Gupta P: TeleCyP (telecytopathology): real-time fine-needle aspiration interpretation. Acta Cytol 2012;56:669-677.

-9 Burlingame OO, Kesse KO, Silverman SG, Cibas ES: On-site adequacy evaluations performed by cytotechnologists: correlation with final interpretations of 5241 image-guided fine-needle aspiration biopsies. Cancer Cytopathol 2012;120:177-184.

10 Wilbur DC: Digital cytology: current state of the art and prospects for the future. Acta $\mathrm{Cy}$ tol 2011;55:227-238.

11 Thrall M, Pantanowitz L, Khalbuss W: Telecytology: clinical applications, current challenges, and future benefits. J Pathol Inform 2011;2:51.

12 Prosch H, Hoffmann E, Bernhardt K, Schalleschak J, Schober E, Rowhani M, Weber M, Mostbeck G: Dynamic telecytologic evaluation of imprint cytology samples from CTguided lung biopsies: a feasibility study. Eur Radiol 2011;21:1922-1927.

13 Khurana KK, Swati I, Kasturi R, Lambert R, Izquierdo R: Telecytopathology for rapid preliminary diagnosis of ultrasound-guided fineneedle aspiration of thyroid nodules. Telemed J E Health 2011;17:763-767.

14 Khalbuss WE, Pantanowitz L, Parwani AV: Digital imaging in cytopathology. Pathol Res Int 2011;2011:264683.

15 Kaplan KJ: Telecytopathology for immediate evaluation of fine-needle aspiration specimens. Cancer Cytopathol 2010;118:115-118.

16 Giovagnoli MR, Giarnieri E, Carico E, Giansanti D: How do young and senior cytopathologists interact with digital cytology? Ann Ist Super Sanita 2010;46:123-129.

17 Giansanti D, Cerroni F, Amodeo R, Filoni M, Giovagnoli MR: A pilot study for the integration of cytometry reports in digital cytology telemedicine applications. Ann Ist Super Sanita 2010;46:138-143.

18 Alsharif M, Carlo-Demovich J, Massey C, Madory JE, Lewin D, Medina AM, Recavarren R, Houser PM, Yang J: Telecytopathology for immediate evaluation of fine-needle aspiration specimens. Cancer Cytopathol 2010; 118:119-126.

19 Pinco J, Goulart RA, Otis CN, Garb J, Pantanowitz L: Impact of digital image manipulation in cytology. Arch Pathol Lab Med 2009; 133:57-61.
20 Pantanowitz L, Hornish M, Goulart RA: The impact of digital imaging in the field of cytopathology. Cytojournal 2009;6:6.

21 Archondakis S, Georgoulakis J, Stamataki M, Anninos D, Skagias L, Panayiotides I, Peros G, Karakitsos P: Telecytology: a tool for quality assessment and improvement in the evaluation of thyroid fine-needle aspiration specimens. Telemed J E Health 2009; 15:713717.

22 Kerr SE, Bellizzi AM, Stelow EB, Frierson HF Jr, Policarpio-Nicolas ML: Initial assessment of fine-needle aspiration specimens by telepathology: validation for use in pathology resident-faculty consultations. Am J Clin Pathol 2008;130:409-413.

23 Eichhorn JH, Buckner L, Buckner SB, Beech DP, Harris KA, McClure DJ, Crothers BA, Wilbur DC: Internet-based gynecologic telecytology with remote automated image selection: results of a first-phase developmental trial. Am J Clin Pathol 2008;129:686-696.

$\checkmark 24$ Burton K, Chhieng D, Jhala N, Jhala D, Winokur T, Crowe D, Eloubeidi M, Eltoum I: Dynamic telecytopathology has equivalent efficacy with on site rapid cytology diagnosis for pancreatic carcinoma. Cancer Cytopathol 2006;108:357-358.

25 Yamashiro K, Kawamura N, Matsubayashi S, Dota K, Suzuki H, Mizushima H, Wakao F, Azumi N: Telecytology in Hokkaido Island, Japan: results of primary telecytodiagnosis of routine cases. Cytopathology 2004;15:221227.

-26 Lee ES, Kim IS, Choi JS, Yeom BW, Kim HK, Han JH, Lee MS, Leong AS: Accuracy and reproducibility of telecytology diagnosis of cervical smears. A tool for quality assurance programs. Am J Clin Pathol 2003;119:356-360.

27 Weinstein RS, Graham AR, Lian F, Braunhut BL, Barker GR, Krupinski EA, Bhattacharyya AK: Reconciliation of diverse telepathology system designs. Historic issues and implications for emerging markets and new applications. APMIS 2012;120:256-275. 
28 Sirintrapun SJ, Cimic A: Dynamic nonrobotic telemicroscopy via skype: a cost effective solution to teleconsultation. J Pathol Inform 2012; 3:28.

29 Gould PV, Saikali S: A comparison of digitized frozen section and smear preparations for intraoperative neurotelepathology. Anal Cell Pathol 2012;35:85-91.

-30 Brachtel E, Yagi Y: Digital imaging in pathology - current applications and challenges. J Biophotonics 2012;5:327-335.

31 Pantanowitz L, Tuthill J, Balis U: Telepathology; in Pantanowitz L, Tuthill J, Balis U (eds): Pathology Informatics: Theory and Practice. Chicago, American Society for Clinical Pathology Press, 2012, pp 257-272.

32 Della Mea V: 25 years of telepathology research: a bibliometric analysis. Diagn Pathol 2011;6(suppl 1):S26.

- 33 Williams S, Henricks WH, Becich MJ, Toscano M, Carter AB: Telepathology for patient care: what am I getting myself into? Adv Anat Pathol 2010;17:130-149.

- 34 Gabril MY, Yousef GM: Informatics for practicing anatomical pathologists: marking a new era in pathology practice. Mod Pathol 2010;23:349-358.
35 Wilbur DC, Madi K, Colvin RB, Duncan LM, Faquin WC, Ferry JA, Frosch MP, Houser SL, Kradin RL, Lauwers GY, Louis DN, Mark EJ, Mino-Kenudson M, Misdraji J, Nielsen GP, Pitman MB, Rosenberg AE, Smith RN, Sohani AR, Stone JR, Tambouret RH, Wu CL, Young RH, Zembowicz A, Klietmann W: Wholeslide imaging digital pathology as a platform for teleconsultation: a pilot study using paired subspecialist correlations. Arch Pathol Lab Med 2009;133:1949-1953.

36 Weinstein RS, Graham AR, Richter LC, Barker GP, Krupinski EA, Lopez AM, Erps KA, Bhattacharyya AK, Yagi Y, Gilbertson JR: Overview of telepathology, virtual microscopy, and whole slide imaging: prospects for the future. Hum Pathol 2009;40:1057-1069.

37 Lopez AM, Graham AR, Barker GP, Richter LC, Krupinski EA, Lian F, Grasso LL, Miller A, Kreykes LN, Henderson JT, Bhattacharyya AK, Weinstein RS: Virtual slide telepathology enables an innovative telehealth rapid breast care clinic. Hum Pathol 2009;40:1082-1091.

38 Leung ST, Kaplan KJ: Medicolegal aspects of telepathology. Hum Pathol 2009;40:11371142.

-39 Krupinski EA: Virtual slide telepathology workstation-of-the-future: lessons learned from teleradiology. Semin Diagn Pathol 2009; 26:194-205.
0 Dee FR: Virtual microscopy in pathology education. Hum Pathol 2009;40:1112-1121.

41 Bellina L, Missoni E: Mobile cell-phones (Mphones) in telemicroscopy: increasing connectivity of isolated laboratories. Diagn Pathol 2009;4:19.

42 Kumar S, Dunn B: Telecytology: a retrospect and prospect; in Kumar S, Dunn B (eds): Telepathology. Berlin, Springer, 2009, pp 149162.

43 Kumar S, Dunn B: Introduction to telepathology; in Kumar S, Dunn B (eds): Telepathology. Berlin, Springer, 2009, pp 1-4.

44 Telepathology symposium. Hum Pathol 2009; 40:1057-1212.

45 O'Malley DP: Practical applications of telepathology using morphology-based anatomic pathology. Arch Pathol Lab Med 2008;132: 743-744.

46 Mencarelli R, Marcolongo A, Gasparetto A: Organizational model for a telepathology system. Diagn Pathol 2008;3(suppl 1):S7.

47 Mulford DK: Telepathology education: reaching out to cytopathology programs throughout the country. ASC Bull 2006;43: 25-30. 